OPEN ACCESS

Check for updates
${ }^{1}$ Department of Clinical

Pharmacology, Copenhagen

University Hospital Bispebjerg

and Frederiksberg, Copenhagen

NV, Denmark

${ }^{2}$ Department of Epidemiology

Research, Statens Serum Institut,

Copenhagen S, 2300, Denmark

${ }^{3}$ Faculty of Health and

Medical Sciences, University

of Copenhagen, Copenhagen,

Denmark

Correspondence to:

N W Andersson

nwandersson@gmail.com

(ORCID 0000-0001-7622-6303)

Additional material is published

online only. To view please visit

the journal online.

Cite this as: $B M / 2021 ; 372: n 107$ http://dx.doi.org/10.1136/bmi.n107

Accepted: 15 December 2020

\section{Association between use of macrolides in pregnancy and risk of major birth defects: nationwide, register based cohort study}

\author{
Niklas Worm Andersson, ${ }^{1,2}$ Rasmus Huan Olsen, ${ }^{1}$ Jon Trærup Andersen ${ }^{1,3}$
}

\author{
ABSTRACT \\ OBJECTIVE \\ To examine the association between the use of \\ macrolide antibiotics in pregnancy and the risk of \\ major birth defects. \\ DESIGN \\ Nationwide, register based cohort study. \\ SETTING \\ Denmark, 1997-2016. \\ PARTICIPANTS
}

Of 1192539 live birth pregnancies, pregnancies during which macrolides had been used (13019) were compared with those during which penicillin (that is, phenoxymethylpenicillin) had been used (matched in a 1:1 ratio on propensity scores). Other comparative groups were pregnancies when macrolides had been used recently but before pregnancy (matched 1:1) and pregnancies where no antibiotics had been used (matched 1:4).

\section{MAIN OUTCOME MEASURES}

Association with an outcome of any major birth defect and specific subgroups of birth defects were assessed by relative risk ratios and absolute risk differences.

RESULTS

In matched comparisons, 457 infants were born with major birth defects to women who had used macrolides during pregnancy (35.1 per 1000 pregnancies) compared with 481 infants (37.0 per 1000 pregnancies) to women who had used penicillin (relative risk ratio $0.95 ; 95 \%$ confidence interval 0.84 to 1.08), corresponding to an absolute risk difference of -1.8 (95\% confidence interval -6.4 to 2.7) per 1000 pregnancies. The risk of major birth defects was not significantly increased for women who had used macrolides during pregnancy compared with those who had used macrolides recently but before becoming pregnant (relative risk ratio 1.00 (95\% confidence interval 0.88 to 1.14 ); absolute

\section{WHAT IS ALREADY KNOWN ON THIS TOPIC}

Macrolides are among the most commonly used antibiotics in pregnancy

Recent findings suggested an increased risk of major birth defects in women who used macrolides compared with those who used penicillin in the first trimester

\section{WHAT THIS STUDY ADDS}

In this large cohort study based on nationwide data of all pregnancies in Denmark (1997-2016) where macrolides were used, no association was found between the use of macrolides during pregnancy and the risk of major birth defects

No evidence was found of an increased risk of organ specific subgroups of birth defects in pregnant women who used macrolides, including defects of the heart, and no evidence of significant associations for individual macrolides risk difference -0.1 (95\% confidence interval -4.8 to 4.7) per 1000 pregnancies) or compared with women who did not use any antibiotics (1.05 (0.95 to 1.17); 1.8 ( -1.7 to 5.3$)$ per 1000 pregnancies). For all three comparative group analyses and in the analyses of use of individual macrolides, no significant increased risk of specific subgroups of birth defects associated with the use of macrolides was found.

\section{CONCLUSIONS}

In this nationwide cohort study, the use of macrolide antibiotics in pregnancy was not associated with an increased risk of major birth defects. Analyses of the associated risk of 12 specific subgroups of birth defects with the use of macrolides in pregnancy were not significant.

\section{Introduction}

Macrolide antibiotics are used for the treatment of common bacterial infections, such as upper and lower respiratory infections and sexually transmitted diseases, and are frequently used as alternatives for patients allergic to penicillin. Macrolides (such as azithromycin, clarithromycin, and erythromycin) are among the most commonly prescribed antibiotics during pregnancy in the United States and in European countries. $^{1-6}$

In a recent cohort study, an increased risk of major birth defects was found among 2170 women who used macrolides in the first trimester compared with women who used penicillin, with an adjusted risk ratio of 1.55 (95\% confidence interval 1.19 to 2.03$){ }^{7}$ Specifically, the study suggested a significantly increased risk of cardiovascular and genital defects with the use of macrolides in the first trimester and in any trimester, respectively. Data from previous studies are insufficient to properly evaluate the potential risk of birth defects overall and the risk of specific subgroups of birth defects among women who used macrolides during pregnancy. ${ }^{8-11}$

Given the widespread use of macrolides, a 1.5fold increased risk would result in excess birth defects that could have been prevented by the use of an alternative antibiotic. Before a change in the clinical recommendations on prescribing macrolides in pregnancy is implemented, however, the findings must be confirmed in other independent populations. In Denmark, macrolides and penicillin (that is, phenoxymethylpenicillin, which is the most commonly used antibiotic in Scandinavia) have a similar range of indications. Although penicillin is generally the first line antibiotic for most of these indications, macrolides are the recommended first alternative for patients who are allergic to penicillin or when a broader spectrum antibiotic is required. We conducted a nationwide 
cohort study in Denmark to examine the association between the use of macrolides in pregnancy and major birth defects compared with the use of penicillin, and compared with other groups of pregnancies where antibiotics were not used.

\section{Methods}

Data sources and study design

We conducted an historical register based cohort study of all recorded pregnancies in Denmark from 1 January 1997 to 31 December 2016. With the unique personal identification number that is assigned to all inhabitants of Denmark, we linked individual level data from different nationwide registries. The Medical Birth Registry has information related to pregnancy for all live birth pregnancies (since 1978). ${ }^{12}$ The National Patient Registry holds information on use of hospital care and diagnoses assigned by the treating physician according to the International Classification of Diseases, version 10 (ICD-10). ${ }^{13}$ We obtained socioeconomic information from the Danish Civil Registration System and Statistics Denmark. ${ }^{14}$ The Registry of Medicinal Product Statistics provided information on all filled prescriptions for macrolides and penicillin and for other drugs from all pharmacies in Denmark. ${ }^{15}$ We estimated the start date of pregnancy from the gestational age registered at the date of birth and subtracting it from the date of birth. Gestational age was calculated from the first day of the last menstrual period and subsequently confirmed by ultrasonography at the antenatal screening. We excluded multiple pregnancy records with overlapping dates and records with implausible or missing information on gestational age. We also excluded pregnancies ending in abortion or miscarriage, and stillbirths.

\section{Use of macrolides and comparative groups}

The use of macrolides was defined as at least one filled prescription for a systemic macrolide antibiotic (anatomical therapeutic chemical (ATC) code J01FA). In Denmark, five different macrolides are available for systemic use: azithromycin, clarithromycin, erythromycin, roxithromycin, and spiramycin. The first day of use was defined by the date of the first filled prescription (index date). The first trimester (start of pregnancy up to and including gestational week 12) was the time window of interest. The main comparative group was women who used systemic phenoxymethylpenicillin (ATC code J01CE02) in the first trimester. Pregnant women were allowed to have sequential filled prescriptions. To minimise the risk of misclassification, we excluded pregnant women who used both macrolides and penicillin in the same pregnancy, and pregnancies where prescriptions for multiple antibiotics were filled on the index date.

Other comparative groups were distinct from the main comparative group and included women who had used macrolides recently, but before becoming pregnant, and women who had not used antibiotics during pregnancy. For the recent use of macrolides group, we included only those pregnancies where women had filled a prescription for a macrolide from six months to one month before becoming pregnant (that is, women had no filled prescriptions for a macrolide in the month before pregnancy or in the first trimester). The second comparative group of pregnancies where no antibiotics were used was defined as pregnant women who did not use any systemic antibiotic (ATC J01, P01AB01) from one month before or in the first trimester of pregnancy.

\section{Outcomes}

Major birth defects, diagnosed during inpatient or outpatient care, were defined as infants diagnosed in the first year of life with major birth defects according to the classification system of the European Surveillance of Congenital Anomalies (EUROCAT) of subgroups of major congenital anomalies. ${ }^{16}$ We excluded major birth defects with known causes and minor defects according to the EUROCAT exclusion list (see supplementary material for definitions).

\section{Covariates}

We used propensity score matching to consider a wide range of baseline characteristics with the intention to isolate the association between the outcomes and the use of macrolides in pregnancy. These included potential confounders, risk factors for major birth defects, or proxies for them. For each comparative study cohort, we performed individual propensity score estimations and matchings. ${ }^{17}{ }^{18}$ Propensity scores were estimated with a logistic regression model and included all variables listed in supplementary table A as predictors. Gestational week when antibiotic treatment was started was included in the propensity scores for the comparative analyses of the use of macrolides versus the use of penicillin. Based on the propensity scores, we created matched cohorts of: women who used macrolides versus those who used penicillin during pregnancy (matched in a 1:1 ratio); women who used macrolides versus those who used macrolides recently, but before becoming pregnant (1:1); and women who used macrolides versus those who did not use any antibiotics during pregnancy (1:4).

\section{Statistical analysis}

Matching was performed with the greedy nearest neighbour matching algorithm (calliper width 0.02 on the propensity score scale). Missing values were low (six variables had missing values, ranging from $0.0 \%$ to $2.6 \%$ for the main comparison cohort; supplementary table B) and imputed with the mode value. The quality of matching was assessed by standardised differences. A covariate with a standardised difference of less than $10 \%$ between matched groups was considered well balanced. The associations were assessed by relative risk ratios and absolute risk differences, with their corresponding 95\% confidence interval, computed with log binomial models. Statistical tests were two sided; effect estimates were considered statistically 
significant if the $95 \%$ confidence interval of the relative estimates did not overlap with 1 and 0 for the absolute estimates. In secondary analyses, we assessed the risk of specific subgroups of birth defects according to organ systems for women who used macrolides during pregnancy compared with those who used penicillin, and compared with the other comparative groups who did not use antibiotics during pregnancy.

In subgroup analyses, we compared the risk of major birth defects and the specific subgroups of birth defects of the individual macrolides (azithromycin, clarithromycin, erythromycin, and roxithromycin) with the respective matched group of women who used penicillin during pregnancy. No correction for multiple testing was applied. Because of national regulations on data protection, we only included analyses where cells of data were three or more. Also, for the main comparison, we performed a subgroup analysis on pregnancies where women started treatment with an antibiotic during gestational weeks 3 to 8 (that is, during organogenesis, when a teratogenic effect generally is considered to be most pronounced, if present, including those that lead to heart defects) ${ }^{19}$ for the outcome of any major birth defect and defects of the heart. We also performed a subgroup analysis on singleton pregnancies. Because the main analyses allowed women to be included in the study multiple times if they had more than one pregnancy during the study period, we performed more sensitivity analyses on first time pregnancies only, and on the first pregnancy only where women used an antibiotic (that is, women could contribute to the analysis only one (the first) pregnancy where they used antibiotics).

Other subgroup analyses for the main comparison were performed where we excluded pregnant women who had used any systemic antibiotic in the month before pregnancy and any other systemic antibiotic in the first trimester. We also analysed the risk of birth defects when we included defects identified in induced abortions after gestational week 12 . We conducted post hoc analyses to assess the association between genital defects and pregnancies where macrolides were used at any time during pregnancy and in the second and third trimesters. We also analysed the risk of individual categories of heart defects to test for positive associations and to expand on previously reported significant findings. ${ }^{7}$

\section{Patient and public involvement}

No patients were involved in setting the research question, study design, outcome measures, or the conduct of the study. The study was based on deidentified historical data from Danish national registries.

\section{Results}

\section{Study cohorts}

The source cohort included 1192539 live birth pregnancies. After we applied the eligibility criteria for each comparative group, 13019 pregnancies during which macrolides had been used, 51515 during which penicillin had been used, 40345 pregnancies when macrolides had been used recently but before pregnancy, and 995673 pregnancies where no antibiotics had been used were eligible for inclusion in the study (fig 1). The median (interquartile range) gestational day for the start of treatment with a macrolide was day 28 (13-54), that is, days since the start of pregnancy (supplementary table C shows unmatched baseline characteristics). Among the pregnancies during which macrolides had been used, 4712 (36.2\%) used azithromycin, 468 (3.6\%) clarithromycin, 5459 (41.9\%) erythromycin, 2375 (18.2\%) roxithromycin, and five $(0.0 \%)$ spiramycin. The propensity score matched cohorts included: 26034 pregnancies for the analysis of use of macrolides versus use of penicillin (matched in a 1:1 ratio); 23816 pregnancies for the analysis of use of macrolides versus recent use of macrolides but before the start of pregnancy (1:1 ratio); and 65094 pregnancies for the analysis of use of macrolides versus no antibiotics used (in an up to 1:4 ratio) (table 1). Supplementary table D shows the number of tablets redeemed and the estimated days of coverage, and supplementary table E shows other pregnancy outcomes. Across all matched cohorts, baseline characteristics were well balanced between the groups with standardised differences of less than 10\% (supplementary table $\mathrm{F}$ and supplementary figure).

\section{Major birth defects}

In the main comparison analysis (use of macrolides $v$ use of penicillin during pregnancy), 457 infants were diagnosed with a major birth defect (35.1 per 1000 pregnancies) in the group where macrolides were used compared with 481 infants $(37.0$ per 1000 pregnancies) in the penicillin group (relative risk ratio 0.95 ; 95\% confidence interval 0.84 to 1.08 ) (fig 2), corresponding to an absolute risk difference of -1.8 (95\% confidence interval -6.4 to 2.7 ) per 1000 pregnancies (supplementary table $\mathrm{G}$ ). Compared with pregnancies when macrolides had been used recently but before pregnancy, 433 infants were diagnosed with a major birth defect (36.4 per 1000 pregnancies) in the group where macrolides were used during pregnancy compared with 434 infants (36.4 per 1000 pregnancies) in the group where women had used macrolides recently but before becoming pregnant (relative risk ratio 1.00; 95\% confidence interval 0.88 to 1.14) (fig 2 ), corresponding to an absolute risk difference of -0.1 (95\% confidence interval -4.8 to 4.7 ) per 1000 pregnancies (supplementary table $\mathrm{H}$ ). Compared with pregnancies where no antibiotics were used, 458 infants were diagnosed with a major birth defect (35.2 per 1000 pregnancies) in the group where macrolides were used compared with 1738 infants (33.4 per 1000 pregnancies) in the group where no antibiotics were used (relative risk ratio 1.05; 95\% confidence interval 0.95 to 1.17) (fig 2), corresponding to an absolute risk difference of 1.8 (95\% confidence interval -1.7 to 5.3) per 1000 pregnancies (supplementary table I). Across the three comparative groups, no significant 
1786813

Pregnancy records identified from January 1997 to December 2016

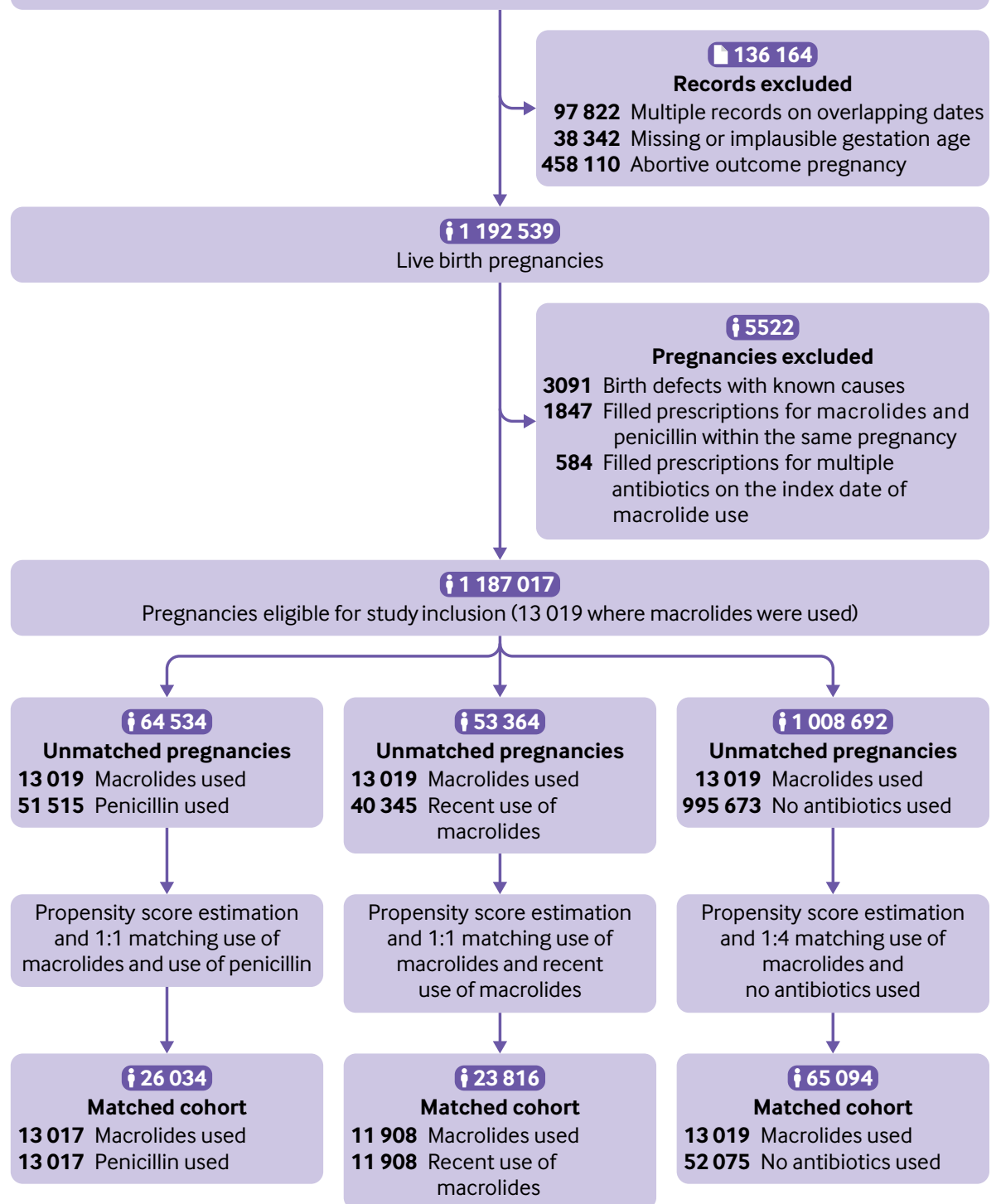

Fig 1 | Flowchart of study cohorts. Cohort selection process for the three comparative cohorts: women who used macrolides during pregnancy versus those who used penicillin (that is, phenoxymethylpenicillin, main comparator); women who used macrolides during pregnancy versus those who used macrolides recently but before becoming pregnant; and women who used macrolides during pregnancy versus those who did not use any antibiotics (additional comparative groups) for the risk of major birth defects

increased risks for any of the specific subgroups of birth defects were identified in pregnancies during which macrolides had been used.

\section{Analyses of individual macrolides}

In analyses performed for individual macrolides (azithromycin, clarithromycin, erythromycin, and roxithromycin), we found no significant associations between the use of individual macrolides during the first trimester and any major birth defects compared with the use of penicillin (fig 3 and supplementary tables J-L). For analyses of the specific subgroups of birth defects, we found no significant associations for any of the individual macrolides.

\section{Sensitivity analyses}

We performed several sensitivity analyses to test the robustness of the main results and to evaluate previously reported significant findings. Compared with pregnancies during which penicillin had been used, we found no association between pregnancies during which macrolides had been used and major birth defects in the subgroups of pregnancies analysed, including when antibiotic treatment was started during gestational weeks 3 to 8 (supplementary table M). Sensitivity analyses including confirmed defects in induced abortions showed similar results (relative risk ratio $0.90 ; 95 \%$ confidence interval 0.77 to 1.05). 
Table 1 | Baseline characteristics of matched pregnancy cohorts on propensity scores

\begin{tabular}{|c|c|c|c|c|c|c|}
\hline \multirow[b]{2}{*}{ Characteristics } & \multicolumn{2}{|c|}{ Macrolides v penicillin } & \multicolumn{2}{|c|}{$\begin{array}{l}\text { Macrolides } v \text { recent } \\
\text { use of macrolides }\end{array}$} & \multicolumn{2}{|c|}{ Macrolides v no antibiotics used } \\
\hline & $\begin{array}{l}\text { Macrolides } \\
(n=13017)\end{array}$ & $\begin{array}{l}\text { Penicillin } \\
(n=13 \text { 017) }\end{array}$ & $\begin{array}{l}\text { Macrolides } \\
(n=11908)\end{array}$ & $\begin{array}{l}\text { Recent use } \\
(n=11908)\end{array}$ & $\begin{array}{l}\text { Macrolides } \\
(n=13019)\end{array}$ & $\begin{array}{l}\text { No antibiotics used } \\
(n=52075)\end{array}$ \\
\hline GA at the start of drug use (median (IQR)) & $28(13-54)$ & $27(12-52)$ & $27(12-52)$ & & $28(13-54)$ & \\
\hline \multicolumn{7}{|l|}{ Age at pregnancy onset } \\
\hline$\leq 19$ & $584(4.5)$ & $453(3.5)$ & $496(4.2)$ & $479(4.0)$ & $586(4.5)$ & $2428(4.7)$ \\
\hline $20-24$ & $2321(17.8)$ & $2267(17.4)$ & $2112(17.7)$ & $2107(17.7)$ & $2321(17.8)$ & $9513(18.3)$ \\
\hline $25-29$ & $3909(30.0)$ & $4051(31.1)$ & $3625(30.4)$ & $3696(31.0)$ & $3909(30.0)$ & $15576(29.9)$ \\
\hline $30-34$ & $3969(30.5)$ & $4038(31.0)$ & $3646(30.6)$ & $3629(30.5)$ & $3969(30.5)$ & $15864(30.5)$ \\
\hline$\geq 35$ & $2234(17.2)$ & $2208(17.0)$ & $2029(17.0)$ & $1997(16.8)$ & $2234(17.2)$ & $8694(16.7)$ \\
\hline Married or living with partner & $10129(77.8)$ & $10282(79.0)$ & $9269(77.8)$ & $9356(78.6)$ & $10129(77.8)$ & $40548(77.9)$ \\
\hline \multicolumn{7}{|l|}{ Place of birth } \\
\hline Denmark & $11475(88.2)$ & $11514(88.5)$ & $10593(89.0)$ & $10727(90.1)$ & $11476(88.2)$ & $46278(88.9)$ \\
\hline Europe & $563(4.3)$ & $527(4.1)$ & $491(4.1)$ & $432(3.6)$ & $563(4.3)$ & $2070(4.0)$ \\
\hline Outside of Europe & $979(7.5)$ & $976(7.5)$ & $824(6.9)$ & 749 (6.3) & $980(7.5)$ & $3727(7.2)$ \\
\hline \multicolumn{7}{|l|}{ Region of residence } \\
\hline Capital Region of Denmark & $4241(32.6)$ & $4312(33.1)$ & $3822(32.1)$ & $3934(33.0)$ & $4241(32.6)$ & $16994(32.6)$ \\
\hline Region Zealand & $2075(15.9)$ & $2040(15.7)$ & $1917(16.1)$ & $1899(16.0)$ & $2075(15.9)$ & $8345(16.0)$ \\
\hline Region of Southern Denmark & $2870(22.1)$ & $2948(22.7)$ & $2645(22.2)$ & $2634(22.1)$ & $2871(22.1)$ & $11492(22.1)$ \\
\hline Central Denmark Region & $2588(19.9)$ & $2537(19.5)$ & $2380(20.0)$ & $2342(19.7)$ & 2589 (19.9) & $10448(20.1)$ \\
\hline North Denmark Region & $1243(9.6)$ & $1180(9.1)$ & $1144(9.6)$ & $1099(9.2)$ & $1243(9.6)$ & $4796(9.2)$ \\
\hline \multicolumn{7}{|l|}{ Gross household income* } \\
\hline 1 & $4280(32.9)$ & $4095(31.5)$ & $3407(28.6)$ & $3307(27.8)$ & $4636(35.6)$ & $18519(35.6)$ \\
\hline 2 & $3184(24.5)$ & $3204(24.6)$ & $3048(25.6)$ & $2923(24.6)$ & $3292(25.3)$ & $13205(25.4)$ \\
\hline 3 & $2777(21.3)$ & $2857(22.0)$ & $2819(23.7)$ & $2924(24.6)$ & $2691(20.7)$ & $10793(20.7)$ \\
\hline 4 & $2776(21.3)$ & $2861(22.0)$ & $2634(22.1)$ & $2754(23.1)$ & $2400(18.4)$ & $9558(18.4)$ \\
\hline \multicolumn{7}{|l|}{ Education level (years) } \\
\hline$<12$ & $4550(35.0)$ & 4409 (33.9) & $4174(35.1)$ & $4062(34.1)$ & $4552(35.0)$ & $18508(35.5)$ \\
\hline $12-13$ & $1913(14.7)$ & $1927(14.8)$ & $1752(14.7)$ & $1737(14.6)$ & $1913(14.7)$ & $7470(14.3)$ \\
\hline $14-15$ & $3181(24.4)$ & $3259(25.0)$ & $2954(24.8)$ & $2973(25.0)$ & $3181(24.4)$ & $12754(24.5)$ \\
\hline$>15$ & $3373(25.9)$ & $3422(26.3)$ & $3028(25.4)$ & $3136(26.3)$ & $3373(25.9)$ & $13343(25.6)$ \\
\hline \multicolumn{7}{|l|}{ Year of start of pregnancy } \\
\hline $1997-2000$ & $3798(29.2)$ & $3880(29.8)$ & $3468(29.1)$ & $3584(30.1)$ & $3799(29.2)$ & $15336(29.5)$ \\
\hline 2001-2004 & $3211(24.7)$ & $3263(25.1)$ & $2923(24.6)$ & $2845(23.9)$ & $3212(24.7)$ & $12749(24.5)$ \\
\hline $2005-2008$ & 2459 (18.9) & $2438(18.7)$ & $2260(19.0)$ & $2327(19.5)$ & 2459 (18.9) & $9894(19.0)$ \\
\hline 2009-2012 & $2222(17.1)$ & $2160(16.6)$ & $2040(17.1)$ & $1983(16.7)$ & $2222(17.1)$ & $8955(17.2)$ \\
\hline $2013-2016$ & $1327(10.2)$ & $1276(9.8)$ & $1217(10.2)$ & $1169(9.8)$ & $1327(10.2)$ & $5141(9.9)$ \\
\hline \multicolumn{7}{|l|}{ Parity } \\
\hline 1 & $5940(45.6)$ & $5945(45.7)$ & $5474(46.0)$ & $5590(46.9)$ & $6006(46.1)$ & $24690(47.4)$ \\
\hline 2 & $4589(35.3)$ & $4643(35.7)$ & 4151 (34.9) & $4163(35.0)$ & $4525(34.8)$ & $17734(34.1)$ \\
\hline$\geq 3$ & $2488(19.1)$ & $2429(18.7)$ & $2283(19.2)$ & $2155(18.1)$ & $2488(19.1)$ & $9651(18.5)$ \\
\hline Multiple birth pregnancy & $459(3.5)$ & 409 (3.1) & $453(3.8)$ & $638(5.4)$ & $459(3.5)$ & $1429(2.7)$ \\
\hline Smoking during pregnancy & $3378(26.0)$ & $3226(24.8)$ & $3120(26.2)$ & $3017(25.3)$ & $3379(26.0)$ & $13205(25.4)$ \\
\hline Previous pregnancy with major birth defect & $196(1.5)$ & $171(1.3)$ & $181(1.5)$ & $160(1.3)$ & $196(1.5)$ & $607(1.2)$ \\
\hline Antidiabetic drug use in past year & $170(1.3)$ & $163(1.3)$ & $170(1.4)$ & $146(1.2)$ & $170(1.3)$ & $500(1.0)$ \\
\hline Drugs used for IVF in past 3 months & $489(3.8)$ & $457(3.5)$ & $487(4.1)$ & $497(4.2)$ & $489(3.8)$ & $1766(3.4)$ \\
\hline \multicolumn{7}{|l|}{ No of drugs used in past year } \\
\hline $1-2$ & $3937(30.3)$ & $3921(30.1)$ & $3932(33.0)$ & $3705(31.1)$ & $4120(31.7)$ & $16866(32.4)$ \\
\hline $3-4$ & $3443(26.5)$ & $3465(26.6)$ & $3438(28.9)$ & $3476(29.2)$ & $3350(25.7)$ & $13770(26.4)$ \\
\hline$\geq 5$ & $4239(32.6)$ & $4317(33.2)$ & $4219(35.4)$ & $4215(35.4)$ & $3967(30.5)$ & $15296(29.4)$ \\
\hline \multicolumn{7}{|l|}{ No of inpatient hospital admissions in past year } \\
\hline 1 & $1540(11.8)$ & $1491(11.5)$ & $1444(12.1)$ & $1321(11.1)$ & $1540(11.8)$ & $5464(10.5)$ \\
\hline 2 & $354(2.7)$ & $342(2.6)$ & $325(2.7)$ & $286(2.4)$ & $354(2.7)$ & $1131(2.2)$ \\
\hline$\geq 3$ & $112(0.9)$ & $98(0.8)$ & $105(0.9)$ & $92(0.8)$ & $112(0.9)$ & $325(0.6)$ \\
\hline \multicolumn{7}{|l|}{ No of outpatient contacts in past year } \\
\hline 1 & $1822(14.0)$ & $1776(13.6)$ & $1701(14.3)$ & $1541(12.9)$ & $1822(14.0)$ & $6612(12.7)$ \\
\hline 2 & $659(5.1)$ & $618(4.8)$ & $621(5.2)$ & $560(4.7)$ & $659(5.1)$ & $2080(4.0)$ \\
\hline$\geq 3$ & $290(2.2)$ & $271(2.1)$ & $282(2.4)$ & $235(2.0)$ & $290(2.2)$ & $945(1.8)$ \\
\hline
\end{tabular}

$\mathrm{GA}=$ gestational age (days since start of pregnancy), IQR=interquartile range, IVF=in vitro fertilisation. Values are number (\%) unless otherwise stated. Percentages might not total 100 because of rounding.

*Income categorised into quarters.

In post hoc analyses, we found that the use of macrolides at any time during pregnancy, and in the second and third trimesters only, was not associated with an increased risk of major birth defects (relative risk ratio 1.02 ; $95 \%$ confidence interval 0.93 to 1.12 , for use in any trimester) or of birth defects of the external genital organs $(0.93 ; 0.68$ to 1.27 , for use in any trimester; supplementary table N). Our primary analyses did not find an association between use of macrolides during pregnancy and the risk of heart 


\begin{tabular}{|c|c|c|c|c|}
\hline Outcome & Macrolides & $\begin{array}{c}\text { Comparative } \\
\text { group }\end{array}$ & $\begin{array}{c}\text { Relative risk } \\
(95 \% \mathrm{Cl})\end{array}$ & $\begin{array}{c}\text { Relative risk } \\
(95 \% \mathrm{Cl})\end{array}$ \\
\hline Macrolides $v$ penicillin & $\begin{array}{c}(n=13017) \\
\text { No of defects }\end{array}$ & $\begin{array}{c}(n=13017) \\
\text { No of defects }\end{array}$ & & \\
\hline Any major birth defect & 457 & 481 & $-\diamond$ & 0.95 (0.84 to 1.08$)$ \\
\hline \multicolumn{5}{|l|}{ Subgroups of birth defects } \\
\hline Nervous system & 19 & 28 & $\longrightarrow \longrightarrow$ & 0.68 (0.38 to 1.21$)$ \\
\hline Eye & 12 & 21 & $-\diamond-$ & $0.57(0.28$ to 1.16$)$ \\
\hline Face, ear, and neck & 4 & 4 & & $1.00(0.25$ to 4.00$)$ \\
\hline Heart & 127 & 129 & $\rightarrow-$ & $0.98(0.77$ to 1.26$)$ \\
\hline Orofacial cleft & 27 & 24 & $\longrightarrow$ & 1.13 (0.65 to 1.95$)$ \\
\hline Digestive system & 23 & 33 & $\bullet-1$ & 0.70 (0.41 to 1.19$)$ \\
\hline Urinary system & 43 & 38 & $\longrightarrow \bullet$ & $1.13(0.73$ to 1.75$)$ \\
\hline External genital organs & 37 & 42 & $-\longrightarrow$ & 0.88 (0.57 to 1.37$)$ \\
\hline Limbs & 138 & 138 & $\rightarrow-$ & $1.00(0.79$ to 1.26$)$ \\
\hline Musculoskeletal system & 21 & 15 & - & $1.40(0.72$ to 2.71$)$ \\
\hline Respiratory system & 23 & 18 & - & 1.28 (0.69 to 2.37$)$ \\
\hline Other major birth defects & 17 & 24 & $\longrightarrow$ & 0.71 (0.38 to 1.32$)$ \\
\hline Macrolides $v$ recent use & $\begin{array}{c}(n=11908) \\
\text { No of defects }\end{array}$ & $\begin{array}{c}(n=11908) \\
\text { No of defects }\end{array}$ & & \\
\hline Any major birth defect & 433 & 434 & $\rightarrow-$ & $1.00(0.88$ to 1.14$)$ \\
\hline \multicolumn{5}{|l|}{ Subgroups of birth defects } \\
\hline Nervous system & 19 & 10 & & 1.90 (0.88 to 4.08$)$ \\
\hline Eye & 11 & 14 & 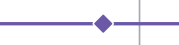 & 0.79 (0.36 to 1.73$)$ \\
\hline Face, ear, and neck & 4 & 5 & & 0.80 (0.21 to 2.98$)$ \\
\hline Heart & 120 & 107 & - & 1.12 (0.87 to 1.45$)$ \\
\hline Orofacial cleft & 26 & 17 & - & 1.53 (0.83 to 2.82$)$ \\
\hline Digestive system & 22 & 24 & $-\bullet$ & $0.92(0.51$ to 1.63$)$ \\
\hline Urinary system & 36 & 35 & 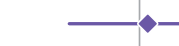 & $1.03(0.65$ to 1.64$)$ \\
\hline External genital organs & 35 & 35 & & $1.00(0.63$ to 1.60$)$ \\
\hline Limbs & 134 & 133 & $\longrightarrow-$ & 1.01 (0.79 to 1.28$)$ \\
\hline Musculoskeletal system & 19 & 22 & - & 0.86 (0.47 to 1.59$)$ \\
\hline Respiratory system & 23 & 20 & - & 1.15 (0.63 to 2.09$)$ \\
\hline Other major birth defects & 17 & 19 & $一-$ & 0.89 (0.47 to 1.72$)$ \\
\hline Macrolides $v$ no antibiotics used & $\begin{array}{c}(n=13019) \\
\text { No of defects }\end{array}$ & $\begin{array}{c}(n=52 \text { 075) } \\
\text { No of defects }\end{array}$ & & \\
\hline Any major birth defect & 458 & 1738 & $-\diamond-$ & $1.05(0.95$ to 1.17$)$ \\
\hline \multicolumn{5}{|l|}{ Subgroups of birth defects } \\
\hline Nervous system & 19 & 69 & $\bullet$ & 1.10 (0.66 to 1.83$)$ \\
\hline Eye & 12 & 54 & $一$ & 0.89 (0.48 to 1.66$)$ \\
\hline Face, ear, and neck & 4 & 20 & & 0.80 (0.27 to 2.34$)$ \\
\hline Heart & 127 & 441 & 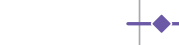 & 1.15 (0.95 to 1.40$)$ \\
\hline Orofacial cleft & 27 & 92 & 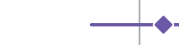 & $1.17(0.76$ to 1.80$)$ \\
\hline Digestive system & 23 & 113 & - & $0.81(0.52$ to 1.27$)$ \\
\hline Urinary system & 43 & 151 & $\longrightarrow-$ & 1.14 (0.81 to 1.60$)$ \\
\hline External genital organs & 37 & 174 & $\rightarrow-$ & $0.85(0.60$ to 1.21$)$ \\
\hline Limbs & 138 & 546 & $\rightarrow-$ & 1.01 (0.84 to 1.22$)$ \\
\hline Musculoskeletal system & 21 & 64 & & 1.31 (0.80 to 2.15$)$ \\
\hline Respiratory system & 24 & 63 & & 1.52 (0.95 to 2.44$)$ \\
\hline \multirow[t]{2}{*}{ Other major birth defects } & 17 & 70 & 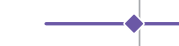 & 0.97 (0.57 to 1.65$)$ \\
\hline & \multicolumn{4}{|c|}{0.1} \\
\hline
\end{tabular}

Fig 2 | Association between the use of macrolides during pregnancy and the risk of major birth defects. The associated risk of major birth defects and specific subgroups of birth defects in women who used macrolides in the first trimester was compared with three distinct propensity score matched pregnancy groups: women who used penicillin (that is, phenoxymethylpenicillin, matched in a 1:1 ratio), women who used macrolides recently but before becoming pregnant (1:1), and women who did not use any antibiotics (1:4) 


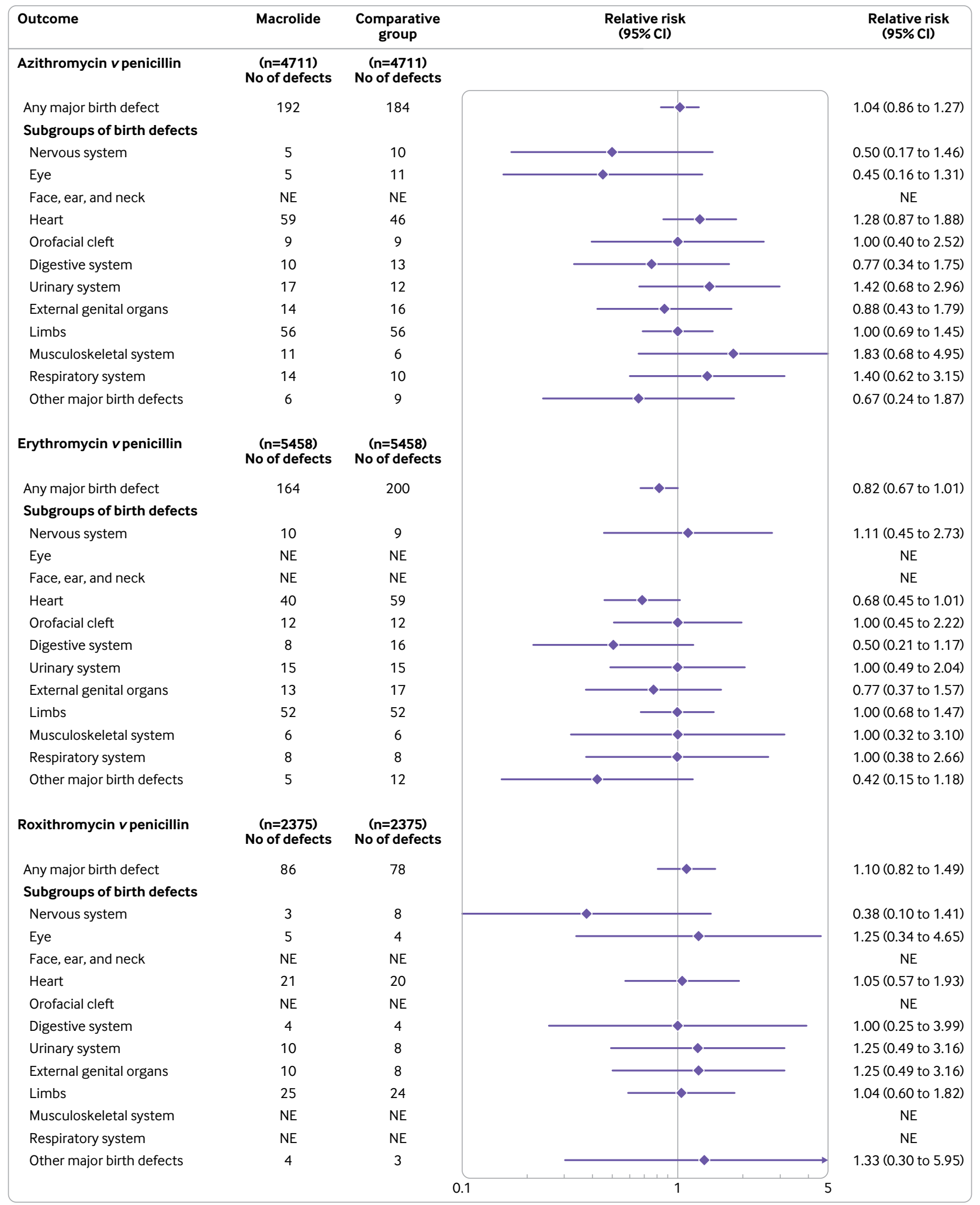

Fig 3 | Associated risk of major birth defects with the use of individual macrolides compared with the use of penicillin in pregnancy. The risk of any major birth defect and specific subgroups of birth defects in women who used individual macrolides in the first trimester were compared with their respective matched women who used penicillin (that is, phenoxymethylpenicillin, matched in a 1:1 ratio). In 468 pregnancies, clarithromycin was used and any major birth defects occurred in 15 pregnancies compared with 19 among pregnancies where penicillin was used (relative risk ratio 0.79; $95 \%$ confidence interval 0.41 to 1.54 ). For the analyses of specific subgroups of birth defects, only heart defects (seven $v$ four, respectively; relative risk ratio $1.75 ; 95 \%$ confidence interval 0.52 to 5.94 ) and limb defects (five $v$ six, respectively; $0.83 ; 0.26$ to 2.71 ) gave a sufficient number of defects for individual analyses. $\mathrm{NE}=$ not estimated because cells of data were less than three 
defects (relative risk ratio $0.98 ; 95 \%$ confidence interval 0.77 to 1.26 compared with use of penicillin during pregnancy, with an absolute risk difference of -0.2 (95\% confidence interval -2.6 to 2.2$)$ per 1000 pregnancies) (fig 2 and supplementary table G). Because a previous study ${ }^{7}$ suggested an increased risk, we performed more post hoc analyses on the association with individual categories of heart defects (table 2). We found no significant associations in any of these analyses.

\section{Discussion}

\section{Principal findings}

In this nationwide cohort study, we found that the use of macrolides in pregnancy was not associated with an increased risk of major birth defects. We included all live birth pregnancies in Denmark where pregnant women had used macrolides during the study period (1997-2016) and compared them with women who had used penicillin and with other comparative groups where antibiotics were not used during pregnancy. Also, our data did not provide evidence of an increased risk of any of the 12 organ specific subgroups of birth defects in women who used macrolides during pregnancy, including defects of the heart.

\section{Interpretation and comparison with previous studies}

This study was prompted by the findings of a recent cohort study which suggested an increased risk of any major birth defect associated with the use of macrolides compared with the use of penicillin in pregnancy (risk ratio 1.55 ; $95 \%$ confidence interval 1.19 to 2.03 ), and specifically cardiovascular defects $(1.62 ; 1.05$ to $2.51) .^{7}$ The study used a clinical database of $6.9 \%$ of the population of the United Kingdom and included 2170 live birth pregnancies where macrolides were used in the first trimester. Our analyses included 13017 pregnancies during which macrolides had been used matched with pregnancies during which penicillin had been used in the first trimester, derived from a nationwide cohort with individual level data obtained from routine clinical care. Our findings did not confirm these previous results and we found no evidence of significant differences in the risk of major birth defects.
Also, we found no associated risk for any of the 12 specific subgroups of birth defects with the use of macrolides in pregnancy. Based on the upper limit of the $95 \%$ confidence intervals from these analyses, a more than twofold relative increase in the risk for nine of the 12 categories can likely be excluded. Also, the results were inconsistent with a more than $26 \%$ relative difference in the risk of heart defects between the use of macrolides and penicillin in pregnancy. Our analyses on the risk of individual categories of heart defects did not show any significant associations. The overall prevalence of heart defects in our study, and in pregnancies where macrolides were used in the first trimester in the UK study, ${ }^{7}$ were in line with the reported prevalence of about $0.9-1.0 \%$ in similar cohorts from the Nordic countries and the US. ${ }^{10}$ 20-22 Differences in the representative samples (probability sampling $v$ nationwide cohort) and other methodological differences between our study and the UK study, such as data collection, might contribute to the different findings. For example, information on birth defects was collected from general practitioner records at the age of three in the UK study (where diagnoses are manually entered into the patient record ${ }^{23}$ ) whereas we obtained data on diagnosed birth defects from inpatient and outpatient hospital care. In Denmark, all diagnoses are automatically recorded in the registries.

Our findings are in line with a recent meta-analysis that included 6082 women who used macrolides during pregnancy from four previously published studies and found no increased risk of birth defects overall (odds ratio 1.03; 95\% confidence interval 0.86 to 1.22$).^{8}$ The meta-analysis did not find any associations with specific subgroups of birth defects, except for gastrointestinal defects (odds ratio 1.56, $95 \%$ confidence interval 1.05 to 2.32 ). This estimate was based on data from one observational cohort study, however, which included 2332 women who used macrolides during pregnancy (most of the results of the meta-analyses on the risk of specific subgroups of birth defects were driven by this one study). ${ }^{11}$ Our study had a much larger cohort of pregnant women who used macrolides than all of these reports combined, thus expanding on previous results by providing estimates with a high precision.

\begin{tabular}{|c|c|c|c|}
\hline \multirow[b]{2}{*}{ Individual categories of heart defect (ICD-10 codes) } & \multicolumn{2}{|c|}{ No of defects (per 1000 pregnancies) } & \multirow[b]{2}{*}{ Relative risk ratio $(95 \% \mathrm{Cl})$} \\
\hline & Macrolides $(n=13017)$ & Penicillin $(n=13017)$ & \\
\hline Defects of cardiac chambers and connections (Q20.0-20.9) & $18(1.4)$ & $11(0.8)$ & $1.64(0.77$ to 3.46$)$ \\
\hline Cardiac septa defects (Q21.0-21.9) & $98(7.5)$ & $106(8.1)$ & 0.92 (0.70 to 1.22$)$ \\
\hline Pulmonary and tricuspid valve defects (Q22.0-22.9) & $16(1.2)$ & $11(0.8)$ & $1.46(0.68$ to 3.13$)$ \\
\hline Aortic and mitral valve defects (Q23.0-23.9) & $10(0.8)$ & $4(0.3)$ & $2.50(0.78$ to 7.97$)$ \\
\hline Other heart defects (Q24.0-24.9) & $22(1.7)$ & $24(1.8)$ & $0.92(0.51$ to 1.63$)$ \\
\hline Defects of the great arteries (Q25.0-25.9) & $12(0.9)$ & $12(0.9)$ & 1.00 (0.45 to 2.23$)$ \\
\hline
\end{tabular}


Our data might not be conclusive for defects that occur rarely, however, and some of our subgroup analyses had limited power. But even if the use of macrolides in pregnancy was associated with a rare defect, given the upper limit of the $95 \%$ confidence interval from the analysis of any major birth defects, our findings are inconsistent with an increase in the relative risk of more than $8 \%$ in pregnant women who used macrolides compared with those who used penicillin. This finding in absolute terms means that, with a reported rate of defects of 37.0 per 1000 pregnancies where penicillin was used, an excess of defects of more than 2.7 per 1000 pregnancies where macrolides were used can likely be ruled out.

Most studies of the associated risk of birth defects with the use of macrolides investigated pregnant women who used erythromycin. Results based on Swedish registry data suggested an association between the use of erythromycin in pregnancy and major birth defects of the heart, ${ }^{21}{ }^{24}$ but this finding has not been replicated in other populations. ${ }^{8} 1025-28$ The results of our study add to previous data as we investigated the associated risk of major birth defects overall, and also of specific subgroups of birth defects, with the use of azithromycin, clarithromycin, erythromycin, and roxithromycin individually. Also, because of the large number of pregnant women who used macrolides in our cohort, we were able to study the risk of individual categories of heart defects. Lastly, our sensitivity analyses, restricted to women who used antibiotics in gestational weeks 3 to 8 , and including defects in induced abortions, did not change the results. To our knowledge, these results have not been previously reported.

We believe that our data provide reassurance about the risk of major birth defects when treatment with macrolide antibiotics is needed during pregnancy. Our findings could help inform clinicians, patients, and drug regulatory authorities.

\section{Strengths and limitations}

Our study cohort was based on all pregnancies in Denmark during the 20 year study period, with individual level data obtained from various nationwide registries, which increased the generalisability of the study findings, and the risk of selection and information bias and loss to follow-up was minimised. In Denmark, all healthcare services are free. The analyses were based on pregnancies resulting in live births only (except for the sensitivity analysis which included defects in induced abortions). Registrations of major birth defects in the National Patient Registry have a high validity, with positive predictive values of $88 \%$ overall and $90 \%$ for heart defects. ${ }^{29} 30$

Data on the specific indication for a filled prescription and adherence are not available from Danish registries. Our definition of use of antibiotics implied that a filled prescription was equivalent to the use of the drug. Thus we cannot exclude differences in adherence in the active comparison analyses, and low adherence in women who used macrolides would bias the results towards the null for all analyses. Although a potential source of confounding for observational studies of drug safety are when the underlying indication being treated increases the risk of an outcome rather than the treatment itself (that is, confounding by indication), we found no significant increased risks in any of our analyses, including compared with other groups who did not use antibiotics during pregnancy. Hence we believe it is unlikely that the indications confounded the associations. To minimise the risk of confounding by indication, however, we used an active comparative design for the main analysis by comparison with the use of phenoxymethylpenicillin during pregnancy, an antibiotic with a similar spectrum of use.

Although a wide range of potential covariates was controlled for in the propensity score, we lacked information on some potentially important confounders from a clinical point of view (such as alcohol consumption, fever, and folic acid supplements) and therefore unmeasured and residual confounding cannot be fully excluded, particularly if unadjusted factors were differently distributed between comparative groups and not controlled for through proxies included in the propensity score. Given the sample size of our study, inherent unadjusted factors masking a true association would have to be common or strongly associated with the use of macrolides and inversely associated with major birth defects among live birth pregnancies. Comparison with other pregnancy groups where antibiotics were not used did not show any significant associations. In summary, we believe the presence of unmeasured confounding factors was limited or unlikely.

\section{Conclusion}

In this nationwide cohort study, we found no significant association between the use of macrolides in the first trimester of pregnancy and major birth defects. In contrast with a recent cohort study from the UK, ${ }^{7}$ we found no significantly increased risk of any of the 12 organ specific subgroups of birth defects, including defects of the heart, and no evidence of significant associations for individual macrolides.

Contributors: NWA had full access to all of the data in the study and takes responsibility for the integrity of the data and the accuracy of the data analyses. All authors conceived and designed the study; acquired, analysed, and interpreted the data; and critically revised the manuscript for important intellectual content. NWA drafted the manuscript and carried out the statistical analysis. NWA and JTA are the guarantors. JTA supervised the study. The corresponding author attests that all listed authors meet authorship criteria and that no others meeting the criteria have been omitted

Funding: The studied received no specific funding.

Competing interests: All authors have completed the ICMJE uniform disclosure form at www.icmje.org/coi disclosure.pdf and declare: no support from any organisation for the submitted work; no financial relationships with any organisations that might have an interest in the submitted work in the previous three years; no other relationships or activities that could appear to have influenced the submitted work. Ethical approval: The study was approved by the Danish Data Protection Agency (2015-41-4309). Ethics approval and informed consent are not required for register based research in Denmark.

Data sharing: No additional data available. 
The lead authors affirm that this manuscript is an honest, accurate, and transparent account of the study being reported; that no important aspects of the study have been omitted; and that any discrepancies from the study as planned (and, if relevant, registered) have been explained.

Dissemination to participants and related patient and public communities: Studied participants were anonymised and deidentified in the utilised data sources; thus the results cannot be directly disseminated to the research participants. The study results will be disseminated to the public and health professionals by a press release, and through social medias with layman's terms.

Provenance and peer review: Not commissioned; externally peer reviewed.

This is an Open Access article distributed in accordance with the Creative Commons Attribution Non Commercial (CC BY-NC 4.0) license, which permits others to distribute, remix, adapt, build upon this work non-commercially, and license their derivative works on different terms, provided the original work is properly cited and the use is non-commercial. See: http://creativecommons.org/licenses/ by-nc/4.0/.

1 Mitchell AA, Gilboa SM, Werler MM, Kelley KE, Louik C, HernándezDíaz S, National Birth Defects Prevention Study. Medication use during pregnancy, with particular focus on prescription drugs: 1976-2008. Am J Obstet Gynecol 2011;205:51.e1-8. doi:10.1016/j. ajog.2011.02.029

2 Andrade SE, Gurwitz JH, Davis RL, et al. Prescription drug use in pregnancy. Am J Obstet Gynecol 2004;191:398-407. doi:10.1016/j. ajog.2004.04.025

3 Engeland A, Bjørge T, Klungsøyr K, Hiellvik V, Skurtveit S, Furu K. Trends in prescription drug use during pregnancy and postpartum in Norway, 2005 to 2015. Pharmacoepidemiol Drug Saf 2018;27:9951004. doi:10.1002/pds. 4577

4 Petersen I, Gilbert R, Evans S, Ridolfi A, Nazareth I. Oral antibiotic prescribing during pregnancy in primary care: UK population-based study. J Antimicrob Chemother 2010;65:2238-46. doi:10.1093/jac/ dkq307

5 de Jonge L, Bos HJ, van Langen IM, de Jong-van den Berg LT, Bakker MK. Antibiotics prescribed before, during and after pregnancy in the Netherlands: a drug utilization study. Pharmacoepidemiol Drug Saf 2014;23:60-8. doi:10.1002/pds.3492

6 Ventura M, Maraschini A, D’Aloja P, et al. Drug prescribing during pregnancy in a central region of Italy, 2008-2012. BMC Public Health 2018:18:623. doi:10.1186/s12889-018-5545-z

7 Fan H, Gilbert R, O'Callaghan F, Li L. Associations between macrolide antibiotics prescribing during pregnancy and adverse child outcomes in the UK: population based cohort study. BMJ 2020;368:m331. doi:10.1136/bmj.m331

8 Fan H, Li L, Wijlaars L, Gilbert RE. Associations between use of macrolide antibiotics during pregnancy and adverse child outcomes: A systematic review and meta-analysis. PLoS One 2019;14:e0212212. doi:10.1371/journal.pone.0212212

9 Einarson A, Phillips E, Mawji F, et al. A prospective controlled multicentre study of clarithromycin in pregnancy. $A m$ I Perinatol 1998;15:523-5. doi:10.1055/s-2007-994053

10 Romøren M, Lindbæk M, Nordeng H. Pregnancy outcome after gestational exposure to erythromycin - a population-based register study from Norway. Br J Clin Pharmacol 2012;74:1053-62. doi:10.1111/j.1365-2125.2012.04286.x

11 Muanda FT, Sheehy O, Bérard A. Use of antibiotics during pregnancy and the risk of major congenital malformations: a population based cohort study. BrJ Clin Pharmacol 2017;83:2557-71. doi:10.1111/ bcp.13364
12 Knudsen LB, Olsen J. The Danish Medical Birth Registry. Dan Med Bull 1998;45:320-3.

13 Lynge E, Sandegaard JL, Rebolj M. The Danish National Patient Register. Scand J Public Health 2011;39(Suppl):30-3. doi:10.1177/1403494811401482

14 Pedersen CB. The Danish Civil Registration System. Scand J Public Health 2011;39(Suppl):22-5. doi:10.1177/1403494810387965

15 Kildemoes HW, Sørensen HT, Hallas J. The Danish National Prescription Registry. Scand J Public Health 2011;39(Suppl):38-41. doi:10.1177/1403494810394717

16 EUROCAT. 1.4. Instructions for the registration and surveillance of congenital anomalies. https://eu-rd-platform.jrc.ec.europa.eu/sites/ default/files/Full Guide 14 _version_28 DEC2018.pdf.

17 Austin PC. Some methods of propensity-score matching had superio performance to others: results of an empirical investigation and Monte Carlo simulations. Biom J 2009;51:171-84. doi:10.1002/ bimj. 200810488

18 Brookhart MA, Wyss R, Layton JB, Stürmer T. Propensity score methods for confounding control in nonexperimental research. Circ Cardiovasc Qual Outcomes 2013;6:604-11. doi:10.1161/ CIRCOUTCOMES.113.000359

19 Sadler TW. Langman's medical embryology. Lippincott Williams \& Wilkins, 2011.

20 Huybrechts KF, Hernández-Díaz S, Straub L, et al. Association of maternal first-trimester ondansetron use with cardiac malformations and oral clefts in offspring. JAMA 2018;320:2429-37. doi:10.1001/ jama.2018.18307

21 Källén BA, Otterblad Olausson P, Danielsson BR. Is erythromycin therapy teratogenic in humans?Reprod Toxicol 2005;20:209-14. doi:10.1016/j.reprotox.2005.01.010

22 Eck LK, Jensen TB, Mastrogiannis D, et al. Risk of adverse pregnancy outcome after paternal exposure to methotrexate within 90 days before pregnancy. Obstet Gynecol 2017;129:707-14. doi:10.1097/ AOG.0000000000001936

23 Herrett E, Gallagher AM, Bhaskaran K, et al. Data resource profile: clinical practice research datalink (CPRD). Int I Epidemiol 2015;44:827-36. doi:10.1093/ije/dyv098

24 Källén B, Danielsson BR, et al. Fetal safety of erythromycin. An update of Swedish data. Eur J Clin Pharmacol 2014;70:355-60. doi:10.1007/s00228-013-1624-3

25 Bahat Dinur A, Koren G, Matok I, et al. Fetal safety of macrolides. Antimicrob Agents Chemother 2013;57:3307-11. doi:10.1128/AAC.01691-12

26 Czeizel AE, Rockenbauer M, Sørensen HT, Olsen J. A population-based case-control teratologic study of oral erythromycin treatment during pregnancy. Reprod Toxicol 1999;13:531-6. doi:10.1016/S08906238(99)00046-5

27 Cooper WO, Hernandez-Diaz S, Arbogast PG, et al. Antibiotics potentially used in response to bioterrorism and the risk of major congenital malformations. Paediatr Perinat Epidemiol 2009;23:1828. doi:10.1111/j.1365-3016.2008.00978x

28 Damkier P, Brønniche LMS, Korch-Frandsen JFB, Broe A. In utero exposure to antibiotics and risk of congenital malformations: a population-based study. Am J Obstet Gynecol 2019;221:648.e1-15. doi:10.1016/j.ajog.2019.06.050

29 Larsen H, Nielsen GL, Bendsen J, Flint C, Olsen J, Sørensen HT. Predictive value and completeness of the registration of congenital abnormalities in three Danish population-based registries. Scand J Public Health 2003;31:12-6. doi:10.1080/14034940210134194

30 Schmidt M, Schmidt SAJ, Sandegaard IL, Ehrenstein V, Pedersen L, Sørensen HT. The Danish National Patient Registry: a review of content, data quality, and research potential. Clin Epidemiol 2015;7:449-90. doi:10.2147/CLEP.S91125

Web appendix: Supplementary material 